\title{
1 TITLE
}

2

3 A low sugar diet enhances Drosophila body size in males and females via sex-specific

4 mechanisms

5

\section{AUTHORS}

7

8 Jason W. Millington ${ }^{1}$, Lianna W. Wat ${ }^{1}$, Ziwei Sun ${ }^{1^{*}}$, Paige J. Basner-Collins ${ }^{1^{*}}$, George P.

9 Brownrigg $^{1^{*}}$, Elizabeth J. Rideout $^{1 \S}$

10

\section{AFFILIATIONS}

12

131 Department of Cellular and Physiological Sciences, Life Sciences Institute, The

14 University of British Columbia, Vancouver, Canada.

15

16 * These authors contributed equally to this work

17

$18 \S$ Corresponding author

19 


\section{RUNNING TITLE}

21

22 Sex-specific mechanisms of low sugar-induced growth

23

24 KEYWORDS

25

26 Drosophila, sex difference, diet, sugar, insulin, target of rapamycin, growth, body size

27

28 CORRESPONDING AUTHOR DETAILS

29

30 Elizabeth J. Rideout, Life Sciences Center, 2350 Health Sciences Mall (RM3308),

31 Vancouver, BC, Canada V6T 1Z3. Email: elizabeth.rideout@ubc.ca Phone: (604) 822-

32 0623. Fax: (604) 822-2316.

33

34 


\section{ABSTRACT}

37 In Drosophila, changes to dietary protein elicit different body size responses between

38 the sexes. Whether this sex difference in nutrient-dependent body size regulation

39 extends to other nutrients, such as dietary sugar, remains unclear. Here, we show that

40 reducing dietary sugar enhanced body size in Drosophila male and female larvae.

41 Indeed, the largest body size was found in larvae reared in a diet without added sugar.

42 Despite the equivalent body size effects of a low sugar diet between males and

43 females, we detected sex-specific changes to the insulin/insulin-like growth factor (IIS)

44 and target of rapamycin (TOR) signaling pathways. Further, we show that the metabolic

45 changes observed in larvae reared on a low sugar diet differ between the sexes. Thus,

46 despite identical phenotypic responses to dietary sugar in males and females, distinct

47 changes to cell signaling pathways and whole-body metabolism were associated with

48 the increased body size in each sex. This highlights the importance of including both

49 sexes in all mechanistic studies on larval growth, as males and females may use

50 different molecular and metabolic mechanisms to achieve similar phenotypic outcomes. 


\section{INTRODUCTION}

54 In Drosophila, dietary nutrients impact the rate and duration of larval growth to influence

55 final body size. Nutrient quantity promotes growth during larval development, as

56 conditions where nutrients are plentiful favour larger body sizes (Edgar, 2006;

57 Hietakangas and Cohen, 2009; Nijhout et al., 2014). Nutrient quality is also critical in

58 regulating larval growth, as individual macronutrients differ in their body size effects. For

59 example, while dietary protein promotes a larger body size across a wide concentration

60 range (Britton and Edgar, 1998; Britton et al., 2002; Edgar, 2006; Shingleton et al.,

61 2017), moderate or high levels of dietary sugar inhibit growth and reduce body size

62 (Musselman et al., 2011; Pasco and Léopold, 2012; Reis, 2016). This suggests a

63 complex relationship between individual macronutrients and body size.

65 Drosophila body size is biological sex (McDonald et al., 2020 preprint; Millington et al.,

66 2021a; Shingleton et al., 2017; Stillwell et al., 2010; Teder and Tammaru, 2005). For

67 example, manipulating nutrient quantity by altering dietary protein and carbohydrates causes sex-biased trait size effects (Shingleton et al., 2017). Male and female

69 phenotypic responses to altered nutrient quality also differ, as the magnitude of protein-

70 dependent changes to body size are larger in females (Millington et al., 2021a). Due to

71 the widespread use of mixed-sex groups in larval growth studies, however, it remains

72 unclear whether sex-specific body size responses to dietary protein extend to other

73 macronutrients, such as sugar. 
75 significantly increased the rate of growth and body size in males and females. Indeed,

76 the largest body size was observed in a diet with no added sugar. Despite the

77 equivalent body size increase in males and females, sex-specific mechanisms underlie

78 the larger body size of larvae raised in a low sugar diet. In females, the low sugar diet

79 stimulated increased target of rapamycin (TOR) pathway activity, whereas the activity of

80 the insulin/insulin-like growth factor signaling pathway (IIS) was enhanced in males.

81 Genetic studies confirmed that these female- and male-specific changes to TOR and

82 IIS, respectively, were important for the low sugar-induced increase in body size, and

83 biochemical studies revealed sex-specific changes to metabolic gene expression and

84 metabolism. Together, our findings provide additional mechanistic insight into how

85 dietary sugar affects development by revealing sex-specific changes to cell signaling

86 pathways and metabolism. This highlights the importance of including both sexes in all

87 larval growth studies, as we show that equivalent phenotypic outcomes may be

88 achieved via distinct mechanisms in each sex.

\section{RESULTS AND DISCUSSION}

A low sugar diet promotes an increased rate of growth and augments body size

94 To determine the body size effects of dietary sugar in each sex, we quantified pupal

95 volume in white ${ }^{1118}$ (w; FBgn0003996) male and female larvae reared in diets with

96 different levels of dietary sugar. Because dietary sugar represses growth in a mixed-sex 
97 larval group (Musselman et al., 2011; Pasco and Léopold, 2012), we started with a 98 widely-used diet (1S) (Lewis, 1960) and removed sugar in a stepwise manner until no

99 added sugar remained (0S). In $w^{1118}$ females, body size was significantly larger in

100 larvae cultured on a diet with half (0.5S), or one-quarter (0.25S), the amount of sugar

101 found in 1S (Fig. 1A). Interestingly, the largest body size was found in female larvae

102 reared in 0S (Fig. 1A).

$103 \quad$ In $w^{1118}$ males, body size was significantly larger in larvae reared on $0.5 S$ and

$1040.25 S$ compared with larvae raised on 1S (Fig. 1B). As in females, the largest body size

105 among males was recorded on 0S (Fig. 1B). Importantly, the body size effects of

106 reduced sugar diets were equivalent between the sexes (Fig. 1C; Supplemental file 4),

107 a finding we reproduced using adult weight (Fig. 1D), indicating that phenotypic

108 responses to dietary sugar were not different between males and females. Because a

109 diet with fewer calories has no effect on body size (Millington et al., 2021a), our findings

110 suggest that the larger size of larvae raised in 0S can be attributed to less dietary sugar.

111 This agrees with data from a mixed-sex larval group showing that dietary sugar inhibits

112 growth (Musselman et al., 2011; Pasco and Léopold, 2012), and extends previous

113 findings by showing the body size effects occur in both sexes.

114 To determine the growth rate in larvae reared on low sugar diets, we measured

115 the time between egg-laying and pupariation in both sexes. In $w^{1118}$ females, time to

$11650 \%$ pupariation was significantly shorter in larvae raised on each reduced-sugar diet

117 compared with genotype-matched larvae cultured in $1 \mathrm{~S}$ (Fig. 1E, F). Time to 50\%

118 pupariation was also reduced in $w^{1118}$ males raised on each reduced-sugar diet

119 compared with genotype-matched males reared on 1S (Fig. 1G, H). Given that diets 
120 with less added sugar shorten the larval growth period and increase body size, our data

121 suggests the larval growth rate in each sex was significantly accelerated in a low sugar

122 context.

123

A low sugar diet has sex-biased effects on insulin/insulin-like growth factor (IIS)

127 Many signaling pathways control organismal and tissue growth during development,

128 however, IIS and TOR have emerged as key regulators of nutrient-dependent growth

129 (Gokhale and Shingleton, 2015; Grewal, 2009; Koyama and Mirth, 2018; Lecuit and Le

130 Goff, 2007; Teleman, 2010). Indeed, high levels of IIS and TOR activity promote a

131 larger body size (Böhni et al., 1999; Britton et al., 2002; Chen et al., 1996; Fernandez et

132 al., 1995; Patel et al., 2003; Poltilove et al., 2000; Zhang et al., 2000). Given the larger

133 body size of males and females cultured on 0S, we examined IIS and TOR activity in

134 larvae reared on 0S and 1S. To measure IIS activity, we quantified mRNA levels of

135 genes that are coregulated by transcription factor Forkhead box, sub-group O (Foxo;

136 FBgn0038197) (e.g. Insulin receptor (InR; FBgn0283499), brummer (bmm; FBgn0036449),

137 and eukaryotic initiation factor 4E-binding protein (4E-BP; FBgn0261560)). For example,

138 when IIS activity is high, Foxo is repressed and mRNA levels of $I n R, b m m$, and $4 E-B P$

139 are low (Alic et al., 2011; Jünger et al., 2003; Puig and Tjian, 2005; Zinke et al., 2002).

$140 \quad$ In $w^{1118}$ females, mRNA levels of Foxo target genes were not different between

141 larvae reared in OS and 1S (Fig. 2A), suggesting IIS activity was not altered in females.

142 In contrast, mRNA levels of Foxo target genes were significantly lower in $w^{1118}$ male 
143 larvae in 0S (Fig. 2B), indicating enhanced IIS activity. Importantly, feeding behaviour

144 was not different between the sexes in either diet (Fig. 2C). While increased IIS activity

145 in males raised on OS may be due to improved insulin sensitivity, changes to mRNA

146 levels of two genes upregulated by insulin insensitivity (Neural Lazarillo (NLaz;

147 FBgn0053126) and puckered (puc; FBgn0243512)) were not consistent with improved

148 insulin sensitivity in either sex (Fig. 2D, E) (Lourido et al., 2021; Pasco and Léopold,

149 2012). Indeed, altered puc mRNA levels in males likely reflect Foxo activity, as puc is a

150 Foxo target (Bai et al., 2013). Together, these findings reveal a previously unrecognized

151 sex difference in IIS regulation in a low sugar context, adding to a growing body of

152 evidence showing sex differences in the nutrient-dependent regulation of IIS in larvae

153 (Millington et al., 2021a).

We next measured TOR activity by monitoring the phosphorylation of TOR's

155 downstream target Ribosomal protein S6 kinase (S6k; FBgn0283472). In $w^{1118}$ females,

156 levels of phosphorylated S6k (pS6k) were higher in 0S in multiple biological replicates

157 (Fig. 2F; Fig S1A), an effect we did not reproduce in $w^{1118}$ males (Fig. 2F; Fig. S1A).

158 This suggests that the low sugar diet caused a female-biased increase in TOR activity,

159 revealing a previously unrecognized sex difference in the nutrient-dependent regulation

160 of TOR. Taken together, these findings not only extend knowledge of sex-specific IIS

161 regulation, but also provide the first report of sex-biased TOR regulation.

163 Sex-biased requirement for IIS, Drosophila insulin-like peptides, and TOR in 
166 To determine whether sex-biased changes to IIS and TOR play a role in mediating the

167 low sugar-induced increase in body size, we measured body size in male and female

168 larvae carrying mutations in each pathway that blunt high levels of IIS and TOR

169 activation (Chen et al., 1996; Millington et al., 2021a; Rideout et al., 2015; Zhang et al.,

170 2000). To determine the requirement for IIS, we measured pupal volume in $w^{1118}$ larvae,

171 and in larvae heterozygous for a hypomorphic allele of $\operatorname{In} R\left(\ln R^{E 19} /+\right)$, in $1 S$ and $0 S$.

172 While body size was larger in $w^{1118}$ male larvae reared on 0S than 1S (Fig. 3A), the low

173 sugar-induced increase in body size was blocked in $\ln R^{E 19} /+$ males (Fig. 3A;

174 genotype:diet interaction $p<0.0001)$. Thus, IIS activity was required in males for

175 increased body size in $0 S$. In contrast, female $w^{1118}$ and $\operatorname{In} R^{E 19} /+$ larvae reared on $0 S$

176 were significantly larger than genotype-matched females raised on 1S (Fig. 3B). While

177 we detected a significant genotype:diet interaction in females $(p<0.0001)$, the magnitude

178 of genotype effects on the body size response to low sugar was smaller in females than

179 in males (sex:diet:genotype interaction $p=0.0114$ ). Thus, reduced IIS function had a

180 male-biased impact on the low sugar-induced increase in body size.

181 Beyond InR, we reared male and female larvae lacking the coding sequences for

182 Drosophila insulin-like peptide 2 (dilp2; Fbgn0036046), Drosophila insulin-like peptide 3

183 (dilp3; Fbgn0044050), and Drosophila insulin-like peptide 5 (dilp5; Fbgn0044038) on 0S

184 and 1S (Grönke et al., 2010). These Dilps are produced and secreted by insulin-

185 producing cells in the brain (Brogiolo et al., 2001; Géminard et al., 2009; Ikeya et al.,

186 2002; Rulifson et al., 2002). Circulating Dilps stimulate IIS activity and growth by binding

187 to InR on target cells (Teleman, 2010). In males, loss of dilp2 and dilp3 blunted the low

188 sugar-induced increase in body compared with $w^{1118}$ controls (genotype:diet $p<0.0001$ 
189 for both); loss of dilp5 had no effect (genotype:diet $p=0.9751)$ (Fig. 3C). In females,

190 while loss of dilp2 and dilp3 (genotype:diet $p<0.0001$ for both), but not dilp5

191 (genotype:diet $p=0.9389)$, blunted the low sugar-induced increase in body size (Fig.

192 3D), the magnitude of genotype effects on the increase in body size were larger in

193 males for dilp3 (sex:diet:genotype interaction: $p=0.0003$ ), with a similar trend in dilp2

194 (sex:diet:genotype interaction: $p=0.0627$ ). Thus, we identify a male-biased requirement

195 for several genes that influence IIS activity in regulating the low sugar-induced increase

196 in body size, a finding that aligns with the male-specific increase in IIS activity in 0 S.

197 To determine the requirement for TOR in mediating the low sugar-induced

198 increase in body size, we measured pupal volume in larvae heterozygous for a

199 hypomorphic allele of Target of rapamycin (Tor; Tor $\left.{ }^{A P} /+\right)\left(\right.$ Zhang et al., 2000). In $w^{1118}$

200 females, larvae reared on 0S were significantly larger than genotype-matched larvae

201 raised on $1 \mathrm{~S}$ (Fig. 3E); however, this low sugar-induced increase in body size was

202 blunted in $\operatorname{Tor}^{\Delta P} /+$ female larvae (Fig. 3E; genotype:diet interaction $\left.p<0.0001\right)$. This

203 suggests the low sugar-induced increase in TOR activity in females was required to

204 achieve a larger body size. In $w^{1118}$ and $\operatorname{Tor}^{A P} /+$ males, pupal volume was significantly

205 larger in larvae raised on 0S compared with genotype-matched larvae reared on $1 S$

206 (Fig. 3F). While the low sugar-induced increase in body size was smaller in $\operatorname{Tor}^{\Delta P} /+$

207 males compared with controls (genotype:diet $p<0.0001$ ), the magnitude of genotype

208 effects on the body size response were larger in females than in males

209 (sex:diet:genotype interaction $(p=0.0303)$. This reveals a previously unrecognized

210 female-biased requirement for TOR activity in regulating body size in a low sugar

211 context. 
213 A low sugar diet has sex-specific effects on metabolic gene expression and

\section{4 whole-body metabolism}

216 IIS and TOR promote increased body size by regulating diverse aspects of metabolism,

217 (e.g. triglyceride storage, protein synthesis, glucose homeostasis) (Grewal, 2009;

218 Musselman and Kühnlein, 2018; Teleman et al., 2008). We therefore measured mRNA

219 levels of a selection of genes implicated in metabolic regulation. In $w^{1118}$ male and

220 female larvae reared on 0S, we found significant changes to mRNA levels compared

221 with larvae reared on 1S, many of which were sex-specific (Fig. 4A). For genes

222 encoding proteins involved in fat metabolism, 9/14 and 5/14 genes showed low sugar-

223 induced changes to mRNA levels in males and females, respectively (Fig. 4A). For

224 genes encoding ribosomal proteins, which play integral roles in protein synthesis, 1/12

225 and 6/12 genes showed low sugar-induced changes to mRNA levels in males and

226 females, respectively (Fig. 4A). While this examination of mRNA levels includes only a

227 fraction of genes that affect metabolism, this data suggests that a low sugar diet causes

228 sex-biased changes to mRNA levels of metabolic genes.

229 To determine the physiological significance of these sex-biased changes in

230 mRNA levels (Gershman et al., 2007; Li et al., 2006; Mattila and Hietakangas, 2017;

231 Teleman et al., 2008; Zinke et al., 2002), we measured whole-body levels of several

232 macronutrients in male and female larvae reared in 0S and 1S. In both male and female

$233 w^{1118}$ larvae reared on 0S, triglyceride levels were significantly reduced compared with

234 sex-matched larvae cultured on 1S (Fig. 4B). Thus, a low sugar diet reduced adiposity 
235 in both sexes. In contrast, $w^{1118}$ females reared in 0S had significantly higher protein

236 levels (Fig. 4C), an effect that was not reproduced in $w^{1118}$ males (Fig. 4C). This reveals

237 a previously unrecognized sex difference in the regulation of whole-body protein in a

238 low sugar context. While glucose levels were significantly higher in $w^{1118}$ male and

239 female larvae raised in 0S (Fig. 4D), the low sugar diet caused a male-specific increase

240 in glycogen and trehalose (Fig. 4E, F). Thus, we observed both sex-specific and non-

241 sex-specific alterations in whole-body carbohydrate levels in a low sugar context,

242 highlighting the importance of including both sexes when studying diet-induced

243 metabolic changes. Indeed, our findings suggest sugar-induced changes to

244 carbohydrate metabolism found in previous studies were possibly driven by effects in

245 males (Musselman et al., 2011; Pasco and Léopold, 2012).

246 In conclusion, our study adds to a growing literature showing sex-specific effects

247 of dietary nutrients on phenotypes such as body size, metabolism, lifespan, and fertility

248 (Green and Extavour, 2014; Hudry et al., 2019; Klepsatel et al., 2020; Millington et al.,

249 2021a; Regan et al., 2016; Wat et al., 2020). Because we show males and females

250 activate distinct signaling pathways and exhibit sex-specific metabolic changes to

251 achieve equivalent phenotypic outcomes, this suggests the absence of a sexually

252 dimorphic phenotype in larval growth studies does not provide sufficient rationale for

253 using single- or mixed-sex groups of animals. Instead, both sexes must be included to

254 draw accurate conclusions regarding the signaling, metabolic, and body size effects of

255 dietary nutrients.

256

257 MATERIALS AND METHODS 
259 Data availability. Original images of pupae are available upon request. Raw values for

260 all data collected and displayed in this manuscript are available in Supplementary File 1.

261 All data necessary for confirming the conclusions of the article are present within the

262 article, figures, tables, and supplementary files.

264 Fly husbandry. Our $1 \mathrm{~S}$ diet consists of $20.5 \mathrm{~g} / \mathrm{L}$ sucrose, $70.9 \mathrm{~g} / \mathrm{L}$ D-glucose, $48.5 \mathrm{~g} / \mathrm{L}$

265 cornmeal, $45.3 \mathrm{~g} / \mathrm{L}$ yeast, $4.55 \mathrm{~g} / \mathrm{L}$ agar, $0.5 \mathrm{~g} \mathrm{CaCl} 2 \cdot 2 \mathrm{H} 2 \mathrm{O}, 0.5 \mathrm{~g} \mathrm{MgSO} \cdot 7 \mathrm{H} 2 \mathrm{O}, 11.77$

$266 \mathrm{~mL}$ acid mix (propionic acid/phosphoric acid). Our 0S diet consists of $48.5 \mathrm{~g} / \mathrm{L}$ cornmeal,

$26745.3 \mathrm{~g} / \mathrm{L}$ yeast, $4.55 \mathrm{~g} / \mathrm{L}$ agar, $0.5 \mathrm{~g} \mathrm{CaCl} 2 \cdot 2 \mathrm{H} 2 \mathrm{O}, 0.5 \mathrm{~g} \mathrm{MgSO} 4 \cdot 7 \mathrm{H} 2 \mathrm{O}, 11.77 \mathrm{~mL}$ acid

268 mix. Details of $0.75 S, 0.5 S$, and $0.25 S$ diets can be found in Supplementary file 2.

269 Larvae were raised at a density of 50 animals per $10 \mathrm{~mL}$ food at $25^{\circ} \mathrm{C}$, and sexed by

270 gonad size. Adult flies were maintained at a density of twenty flies per vial in single-sex

271 groups.

272

273 Fly strains. The following fly strains from the Bloomington Drosophila Stock Center

274 were used: $w^{1118}$ (\#3605), $\operatorname{InR}^{E 19}(\# 9646), \operatorname{Tor}^{\Delta P}(\# 7014)$. Additional fly strains include:

275 dilp2, dilp3, and dilp5 (Grönke et al., 2010). All fly strains were backcrossed for at least

2766 generations, in addition to extensive prior backcrossing (Grönke et al., 2010;

277 Millington et al., 2021a,b). 
279 Body size. Pupal volume and adult weight were measured as previously described

280 (Delanoue et al., 2010; Millington et al., 2021a; Millington et al., 2021b; Rideout et al.,

281 2015).

282

283 Feeding behaviour. Feeding behavior was quantified as number of mouth-hook

284 contractions per $30 \mathrm{~s}$.

285

Developmental timing. Time to pupariation was measured as previously described

287 (Millington et al., 2021a). Time to 50\% pupariation was calculated per replicate and used for quantification and statistical analysis.

Metabolism assays. Each biological replicate consists of ten female or male larvae.

291 Larvae were frozen on dry ice, and homogenized for lipid, protein, glucose, glycogen, 292 and trehalose assays. All assays were performed as described in Tennessen et al. 293 (2014) and Wat et al. (2020).

RNA extraction and cDNA synthesis. RNA extraction and cDNA synthesis were

296 performed as previously described (Marshall et al., 2012; Rideout et al., 2012; Rideout

297 et al., 2015; Wat et al., 2020). Briefly, each biological replicate consists of ten $w^{1118}$

298 larvae frozen on dry ice and stored at $-80^{\circ} \mathrm{C}$. Each experiment contained 3-4 biological

299 replicates per sex, and each experiment was performed at least twice. RNA was

300 extracted using $500 \mu \mathrm{l}$ Trizol (Thermo Fisher Scientific: \#15596018) and precipitated

301 using isopropanol and $75 \%$ ethanol. Pelleted RNA was resuspended in $200 \mu$ molecular 
302 biology grade water (Corning, 46-000-CV) and stored at $-80^{\circ} \mathrm{C}$ until use. For cDNA

303 synthesis, an equal volume of RNA per reaction was DNase-treated and reverse

304 transcribed using the QuantiTect Reverse Transcription Kit (Qiagen, 205314).

305

Quantitative real-time PCR (qPCR). qPCR was performed as previously described

307 (Marshall et al., 2012; Rideout et al., 2012; Rideout et al., 2015; Wat et al., 2020).

308 Primer list in Supplementary file 3.

310 Preparation of protein samples, SDS-PAGE, and Western blotting. Samples were

311 generated as previously described (Millington et al., 2021a). $20 \mu \mathrm{g}$ of protein was

312 loaded per lane, separated on a 12\% SDS-PAGE gel in SDS running buffer, and

313 transferred onto a nitrocellulose membrane (Bio-Rad) for $2 \mathrm{hr}$ at $40 \mathrm{~V}$ on ice.

314 Membranes were incubated for $24 \mathrm{hr}$ in blocking buffer at $4^{\circ} \mathrm{C}(5 \%$ milk or $5 \%$ BSA in

315 TBST $0.1 \%$ ) and subsequently incubated with primary antibodies overnight at $4^{\circ} \mathrm{C}$. Anti-

316 pS6k (\#9209, Cell Signaling), and anti-Actin (\#8432, Santa Cruz) were used at 1:1000.

317 After $3 \times 2$ min washes in $0.1 \%$ TBST, HRP-conjugated secondary antibodies were

318 used at 1:5000 for pS6k (\#65-6120; Invitrogen) and 1:3000 for actin (\#7076; Cell

319 Signaling). Membranes were washed (3 x 2 min, $2 \times 15 \mathrm{~min})$ in $0.1 \%$ TBST, washed $1 \times$

$3205 \mathrm{~min}$ in TBS, and finally Pierce ECL was applied as per manufacturer's instructions

321 (\#32134, Thermo Scientific). 
bioRxiv preprint doi: https://doi.org/10.1101/2021.03.03 433819; this version posted March 4, 2021. The copyright holder for this preprint (which was not certified by peer review) is the author/funder, who has granted bioRxiv a license to display the preprint in perpetuity. It is made available under aCC-BY-NC-ND 4.0 International license.

\section{Statistical analysis. GraphPad Prism (GraphPad Prism version 8.4.3 for Mac OS X)}

324 was used for all statistical tests, and for figure preparation. Full details of statistical tests

325 and $p$-values are listed in Supplementary file 4. 


\section{ACKNOWLEDGEMENTS}

328 We would like to thank Dr. Linda Partridge for sharing the dilp2, dilp3, and dilp5 mutant

329 strains. We thank the Bloomington Drosophila Stock Center (NIH P400D018537) for

330 stocks used in this study. We acknowledge critical resources and information provided

331 by FlyBase (Thurmond et al., 2018); FlyBase is supported by a grant from the National

332 Human Genome Research Institute at the U.S. National Institutes of Health (U41

$333 \underline{H G 000739})$ and by the British Medical Research Council (MR/N030117/1). We

334 acknowledge that our research takes place on the traditional, ancestral, and unceded

335 territory of the Musqueam people; a privilege for which we are grateful.

337 FUNDING

339 Funding for this study was provided by grants to EJR from the Canadian Institutes for

340 Health Research (PJT-153072), Natural Sciences and Engineering Research Council of

341 Canada (NSERC, RGPIN-2016-04249), Michael Smith Foundation for Health Research

342 (16876), and the Canadian Foundation for Innovation (JELF-34879). JWM was

343 supported by a 4-year CELL Fellowship from UBC; LWW was supported by a British

344 Columbia Graduate Scholarship Award, and a 1-year CELL fellowship from UBC; ZS

345 was supported by an NSERC Undergraduate Student Research Award. 


\section{REFERENCES}

348

349

350

351

352

353

354

355

356

357

358

359

360

361

362

363

Alic, N., Hoddinott, M. P., Vinti, G. and Partridge, L. (2011). Lifespan extension by increased expression of the Drosophila homologue of the IGFBP7 tumour suppressor. Aging Cell 10, 137-147.

Bai, H., Kang, P., Hernandez, A. M. and Tatar, M. (2013). Activin Signaling Targeted by Insulin/dFOXO Regulates Aging and Muscle Proteostasis in Drosophila. PLoS Genet $\mathbf{9}$.

Böhni, R., Riesgo-Escovar, J., Oldham, S., Brogiolo, W., Stocker, H., Andruss, B. F., Beckingham, K. and Hafen, E. (1999). Autonomous control of cell and organ size by CHICO, a Drosophila homolog of vertebrate IRS1-4. Cell 97, 865-75.

Britton, J. S. and Edgar, B. A. (1998). Environmental control of the cell cycle in Drosophila: nutrition activates mitotic and endoreplicative cells by distinct mechanisms. Development 125, 2149-58.

Britton, J. S., Lockwood, W. K., Li, L., Cohen, S. M. and Edgar, B. A. (2002). Drosophila's insulin/PI3-kinase pathway coordinates cellular metabolism with nutritional conditions. Dev Cell 2, 239-49.

Brogiolo, W., Stocker, H., Ikeya, T., Rintelen, F., Fernandez, R. and Hafen, E. (2001). An evolutionarily conserved function of the Drosophila insulin receptor and insulin-like peptides in growth control. Curr Biol 11, 213-21.

Chen, C., Jack, J. and Garofalo, R. S. (1996). The Drosophila insulin receptor is required for normal growth. Endocrinology 137, 846-56.

Delanoue, R., Slaidina, M. and Léopold, P. (2010). The steroid hormone ecdysone controls systemic growth by repressing dMyc function in Drosophila fat cells. Dev Cell 18, 1012-21.

Edgar, B. A. (2006). How flies get their size: genetics meets physiology. Nat Rev Genet 7, 907-16.

Fernandez, R., Tabarini, D., Azpiazu, N., Frasch, M. and Schlessinger, J. (1995). The Drosophila insulin receptor homolog: a gene essential for embryonic development encodes two receptor isoforms with different signaling potential. EMBO J 14, 3373-84.

Géminard, C., Rulifson, E. J. and Léopold, P. (2009). Remote control of insulin secretion by fat cells in Drosophila. Cell Metab 10, 199-207.

Gershman, B., Puig, O., Hang, L., Peitzsch, R. M., Tatar, M. and Garofalo, R. S. (2007). High-resolution dynamics of the transcriptional response to nutrition in Drosophila: a key role for dFOXO. Physiological Genomics 29, 24-34. 
Gokhale, R. H. and Shingleton, A. W. (2015). Size control: the developmental physiology of body and organ size regulation. WIREs Developmental Biology 4, 335-356.

Green, D. A. and Extavour, C. G. (2014). Insulin signalling underlies both plasticity and divergence of a reproductive trait in Drosophila. Proceedings of the Royal Society B: Biological Sciences 281, 20132673.

Grewal, S. S. (2009). Insulin/TOR signaling in growth and homeostasis: a view from the fly world. Int J Biochem Cell Biol 41, 1006-10.

Grönke, S., Clarke, D. F., Broughton, S., Andrews, T. D. and Partridge, L. (2010). Molecular evolution and functional characterization of Drosophila insulin-like peptides. PLoS Genet 6, e1000857.

Hietakangas, V. and Cohen, S. M. (2009). Regulation of tissue growth through nutrient sensing. Annu Rev Genet 43, 389-410.

Hudry, B., de Goeij, E., Mineo, A., Gaspar, P., Hadjieconomou, D., Studd, C., Mokochinski, J. B., Kramer, H. B., Plaçais, P.-Y., Preat, T., et al. (2019). Sex Differences in Intestinal Carbohydrate Metabolism Promote Food Intake and Sperm Maturation. Cell 178, 901-918.e16.

Ikeya, T., Galic, M., Belawat, P., Nairz, K. and Hafen, E. (2002). Nutrient-dependent expression of insulin-like peptides from neuroendocrine cells in the CNS contributes to growth regulation in Drosophila. Curr Biol 12, 1293-300.

Jünger, M. A., Rintelen, F., Stocker, H., Wasserman, J. D., Végh, M., Radimerski, T., Greenberg, M. E. and Hafen, E. (2003). The Drosophila Forkhead transcription factor FOXO mediates the reduction in cell number associated with reduced insulin signaling. $J$ Biol 2, 20.

Klepsatel, P., Knoblochová, D., Girish, T. N., Dircksen, H. and Gáliková, M. (2020). The influence of developmental diet on reproduction and metabolism in Drosophila. BMC Evolutionary Biology 20, 93.

Koyama, T. and Mirth, C. K. (2018). Unravelling the diversity of mechanisms through which nutrition regulates body size in insects. Curr Opin Insect Sci 25, 1-8.

Lecuit, T. and Le Goff, L. (2007). Orchestrating size and shape during morphogenesis. Nature 450, 189-192.

Lewis, E. B. (1960). A new standard food medium. Drosophila Information Service 34, $117-118$. 
Li, M. V., Chang, B., Imamura, M., Poungvarin, N. and Chan, L. (2006). GlucoseDependent Transcriptional Regulation by an Evolutionarily Conserved GlucoseSensing Module. Diabetes 55, 1179-1189.

Lourido, F., Quenti, D., Salgado-Canales, D. and Tobar, N. (2021). Domeless receptor loss in fat body tissue reverts insulin resistance induced by a high-sugar diet in Drosophila melanogaster. Sci Rep 11, 3263.

Marshall, L., Rideout, E. J. and Grewal, S. S. (2012). Nutrient/TOR-dependent regulation of RNA polymerase III controls tissue and organismal growth in Drosophila. EMBO J 31, 1916-30.

Mattila, J. and Hietakangas, V. (2017). Regulation of Carbohydrate Energy Metabolism in Drosophila melanogaster. Genetics 207, 1231-1253.

McDonald, J. M. C., Nabili, P., Thorsen, L., Jeon, S. and Shingleton, A. (2020). Sexspecific plasticity and the nutritional geometry of insulin-signaling gene expression in Drosophila melanogaster. bioRxiv 2020.11.16.385708.

Millington, J. W., Brownrigg, G. P., Chao, C., Sun, Z., Basner-Collins, P. J., Wat, L. W., Hudry, B., Miguel-Aliaga, I. and Rideout, E. J. (2021a). Female-biased upregulation of insulin pathway activity mediates the sex difference in Drosophila body size plasticity. eLife 10, e58341.

Millington, J. W., Brownrigg, G. P., Basner-Collins, P. J., Sun, Z. and Rideout, E. J. (2021b). Genetic manipulation of insulin/insulin-like growth factor signaling pathway activity has sex-biased effects on Drosophila body size. G3 Genes|Genomes|Genetics.

Musselman, L. P. and Kühnlein, R. P. (2018). Drosophila as a model to study obesity and metabolic disease. J Exp Biol 221,.

Musselman, L. P., Fink, J. L., Narzinski, K., Ramachandran, P. V., Hathiramani, S. S., Cagan, R. L. and Baranski, T. J. (2011). A high-sugar diet produces obesity and insulin resistance in wild-type Drosophila. Disease Models \& Mechanisms 4, 842-849.

Nijhout, H. F., Riddiford, L. M., Mirth, C., Shingleton, A. W., Suzuki, Y. and Callier, V. (2014). The developmental control of size in insects. Wiley Interdiscip Rev Dev Biol 3, 113-34.

Pasco, M. Y. and Léopold, P. (2012). High Sugar-Induced Insulin Resistance in Drosophila Relies on the Lipocalin Neural Lazarillo. PLOS ONE 7, e36583.

Patel, P. H., Thapar, N., Guo, L., Martinez, M., Maris, J., Gau, C.-L., Lengyel, J. A. and Tamanoi, F. (2003). Drosophila Rheb GTPase is required for cell cycle progression and cell growth. Journal of Cell Science 116, 3601-3610. 
Poltilove, R. M., Jacobs, A. R., Haft, C. R., Xu, P. and Taylor, S. I. (2000). Characterization of Drosophila insulin receptor substrate. J Biol Chem $\mathbf{2 7 5}$, 23346-54.

Puig, O. and Tjian, R. (2005). Transcriptional feedback control of insulin receptor by dFOXO/FOXO1. Genes Dev 19, 2435-46.

Regan, J. C., Khericha, M., Dobson, A. J., Bolukbasi, E., Rattanavirotkul, N. and Partridge, L. (2016). Sex difference in pathology of the ageing gut mediates the greater response of female lifespan to dietary restriction. Elife 5, e10956.

Reis, T. (2016). Effects of Synthetic Diets Enriched in Specific Nutrients on Drosophila Development, Body Fat, and Lifespan. PLOS ONE 11, e0146758.

Rideout, E. J., Marshall, L. and Grewal, S. S. (2012). Drosophila RNA polymerase III repressor Maf1 controls body size and developmental timing by modulating tRNAiMet synthesis and systemic insulin signaling. Proc Natl Acad Sci U S A 109, 1139-44.

Rideout, E. J., Narsaiya, M. S. and Grewal, S. S. (2015). The Sex Determination Gene transformer Regulates Male-Female Differences in Drosophila Body Size. PLoS Genet 11, e1005683.

Rulifson, E. J., Kim, S. K. and Nusse, R. (2002). Ablation of insulin-producing neurons in flies: growth and diabetic phenotypes. Science 296, 1118-20.

Shingleton, A. W., Masandika, J. R., Thorsen, L. S., Zhu, Y. and Mirth, C. K. (2017). The sex-specific effects of diet quality versus quantity on morphology in Drosophila melanogaster. Royal Society Open Science 4, 170375.

Stillwell, R. C., Blanckenhorn, W. U., Teder, T., Davidowitz, G. and Fox, C. W. (2010). Sex Differences in Phenotypic Plasticity Affect Variation in Sexual Size Dimorphism in Insects: From Physiology to Evolution. Annual Review of Entomology 55, 227-245.

Teder, T. and Tammaru, T. (2005). Sexual size dimorphism within species increases with body size in insects. Oikos 108, 321-334.

Teleman, A. A. (2010). Molecular mechanisms of metabolic regulation by insulin in Drosophila. Biochemical Journal 425, 13-26.

Teleman, A. A., Hietakangas, V., Sayadian, A. C. and Cohen, S. M. (2008). Nutritional control of protein biosynthetic capacity by insulin via Myc in Drosophila. Cell Metab 7, 21-32.

Tennessen, J. M., Barry, W., Cox, J. and Thummel, C. S. (2014). Methods for studying metabolism in Drosophila. Methods 68, 105-115. 
Thurmond, J., Goodman, J. L., Strelets, V. B., Attrill, H., Gramates, L. S., Marygold, S. J., Matthews, B. B., Millburn, G., Antonazzo, G., Trovisco, V., et al. (2018). FlyBase 2.0: the next generation. Nucleic Acids Research 47, D759-D765.

Wat, L. W., Chao, C., Bartlett, R., Buchanan, J. L., Millington, J. W., Chih, H. J., Chowdhury, Z. S., Biswas, P., Huang, V., Shin, L. J., et al. (2020). A role for triglyceride lipase brummer in the regulation of sex differences in Drosophila fat storage and breakdown. PLOS Biol 18, e3000595.

Zhang, H., Stallock, J. P., Ng, J. C., Reinhard, C. and Neufeld, T. P. (2000). Regulation of cellular growth by the Drosophila target of rapamycin dTOR. Genes Dev 14, 2712-2724.

Zinke, I., Schütz, C. S., Katzenberger, J. D., Bauer, M. and Pankratz, M. J. (2002). Nutrient control of gene expression in Drosophila: microarray analysis of starvation and sugar-dependent response. EMBO J 21, 6162-73. 
Figure 1. A low sugar diet promotes an increased rate of growth and final body

512 Pupal volume in $w^{1118}$ males cultured on 1S, 0.75S, 0.5S, 0.25S, and 0S. (C) Reaction

513 norms for pupal volume in both sexes plotted using $1 \mathrm{~S}$ and $0 \mathrm{~S}$ data from $\mathrm{A}$ and $\mathrm{B}$. (D)

514 Adult weight in $w^{1118}$ female and male flies reared on $1 \mathrm{~S}$ and $0 \mathrm{~S}$. $(\mathrm{E}, \mathrm{F})$ Time to

515 pupariation in $w^{1118}$ females cultured on $1 S, 0.75 S, 0.5 S, 0.25 S$, and $0 S$. (G, H) Time to

516 pupariation in $w^{1118}$ males cultured on $1 S, 0.75 S, 0.5 S, 0.25 S$, and $0 S .{ }^{*} p<0.05 ;$ **

$517 p<0.01 ;{ }^{* * *} p<0.001 ;{ }^{* * *} p<0.0001 ;$ ns indicates not significant; error bars indicate SEM;

518 dashed lines indicate 95\% confidence interval. $p$-values, samples sizes, and statistical

519 tests are in Supplementary file 4.

521 Figure 2. A low sugar diet has sex-biased effects on insulin/insulin-like growth

522 factor (IIS) and target of rapamycin (TOR) signaling. (A) mRNA levels of Foxo target

523 genes $(\mathrm{InR}, \mathrm{bmm}$, and $4 E-B P)$ in female larvae reared on $1 \mathrm{~S}$ or $0 \mathrm{~S}$. (B) mRNA levels of

524 Foxo target genes in male larvae reared on 1S or 0S. (C) Mouth-hook contractions in

$525 w^{1118}$ female and male larvae raised on $1 S$ or 0S. (D) mRNA levels of NLaz and puc in

526 female larvae raised in $1 \mathrm{~S}$ or $0 \mathrm{~S}$. (E) mRNA levels of NLaz and puc in male larvae

527 raised in 1S or 0S. (F) Levels of phosphorylated S6 kinase (pS6k) in females and males

528 raised in $1 \mathrm{~S}$ or $0 \mathrm{~S} .{ }^{* *} \mathrm{p}<0.01 ;{ }^{* * *} \mathrm{p}<0.001$; ns indicates not significant; error bars

529 indicate SEM. $p$-values, samples sizes, and statistical tests are in Supplementary file 4. 
531 Figure 3. Sex-biased requirement for IIS, Drosophila insulin-like peptides, and

532 target of rapamycin (TOR) in promoting the low sugar-induced increase in body

533 size. (A) Pupal volume in $w^{1118}$ and $\ln R^{E 19} /+$ males cultured on $1 S$ or 0S. (B) Pupal

534 volume in $w^{1118}$ and $\ln R^{E 19} /+$ females cultured on $1 S$ or 0 S. (C) Pupal volume in $w^{1118,}$

535 dilp2 mutant, dilp3 mutant, and dilp5 mutant males reared on 1S or 0S. (D) Pupal

536 volume in $w^{1118}$, dilp2 mutant, dilp3 mutant, and dilp5 mutant females reared on $1 \mathrm{~S}$ or

537 0S. (E) Pupal volume in $w^{1118}$ and $\operatorname{Tor}^{\triangle P} /+$ females cultured on $1 S$ or $0 S$. (F) Pupal

538 volume in $w^{1118}$ and $\operatorname{Tor}^{\Delta P} /+$ males cultured on $1 \mathrm{~S}$ or $0 \mathrm{~S} .{ }^{*} \mathrm{p}<0.05 ;{ }^{* * *} \mathrm{p}<0.001 ;{ }^{* * * *}$

$539 \quad p<0.0001$; ns indicates not significant; error bars indicate SEM. $p$-values, samples sizes,

540 and statistical tests are in Supplementary file 4. Note: parallel collection of multiple

541 genotypes and diets means that $w^{1118}$ control data in 0S and 1S are the same in Fig.

542 1A, B, 3A-D.

544 Figure 4. A low sugar diet has sex-biased effects on metabolic gene expression

545 and metabolism. (A) mRNA levels of metabolic genes in $w^{1118}$ male and female larvae

546 raised on 15 or 0 S. (B) Whole-body triglyceride levels in $w^{1118}$ female and male larvae

547 reared on $1 S$ or 0 S. (C) Whole-body protein levels in $w^{1118}$ female and male larvae

548 raised on 15 or 0 S. (D) Whole-body glucose levels in $w^{1118}$ female and male larvae

549 cultured on $1 S$ or 0 S. (E) Whole-body glycogen levels in $w^{1118}$ female and male larvae

550 reared on 15 or $0 S$. (F) Whole-body trehalose levels in $w^{1118}$ female and male larvae

551 cultured on 1S or 0S. ${ }^{* *} p<0.001 ;{ }^{* * *} p<0.0001$; ns indicates not significant; error bars

552 indicate SEM. p-values, samples sizes, and statistical tests are in Supplementary file 4. 
bioRxiv preprint doi: https://doi.org/10.1101/2021.03.03.433819; this version posted March 4, 2021. The copyright holder for this preprint (which was not certified by peer review) is the author/funder, who has granted bioRxiv a license to display the preprint in perpetuity. It is made available under aCC-BY-NC-ND 4.0 International license.

554 Figure S1. A low sugar diet has sex-biased effects on target of rapamycin (TOR)

555 signaling. (A) Levels of pS6k in females and males raised in $1 \mathrm{~S}$ or $0 \mathrm{~S}$.

556 

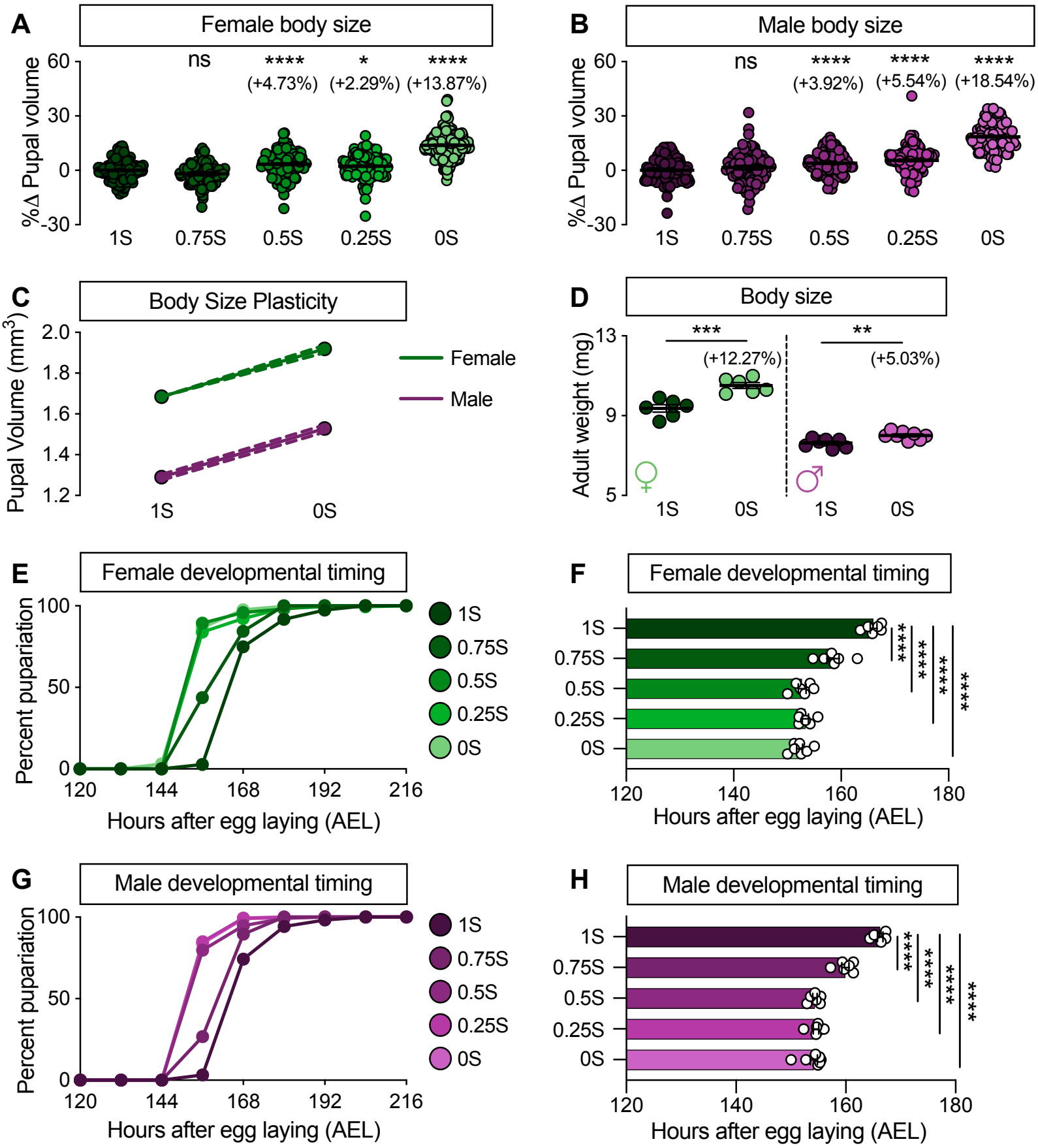
Figure 2.
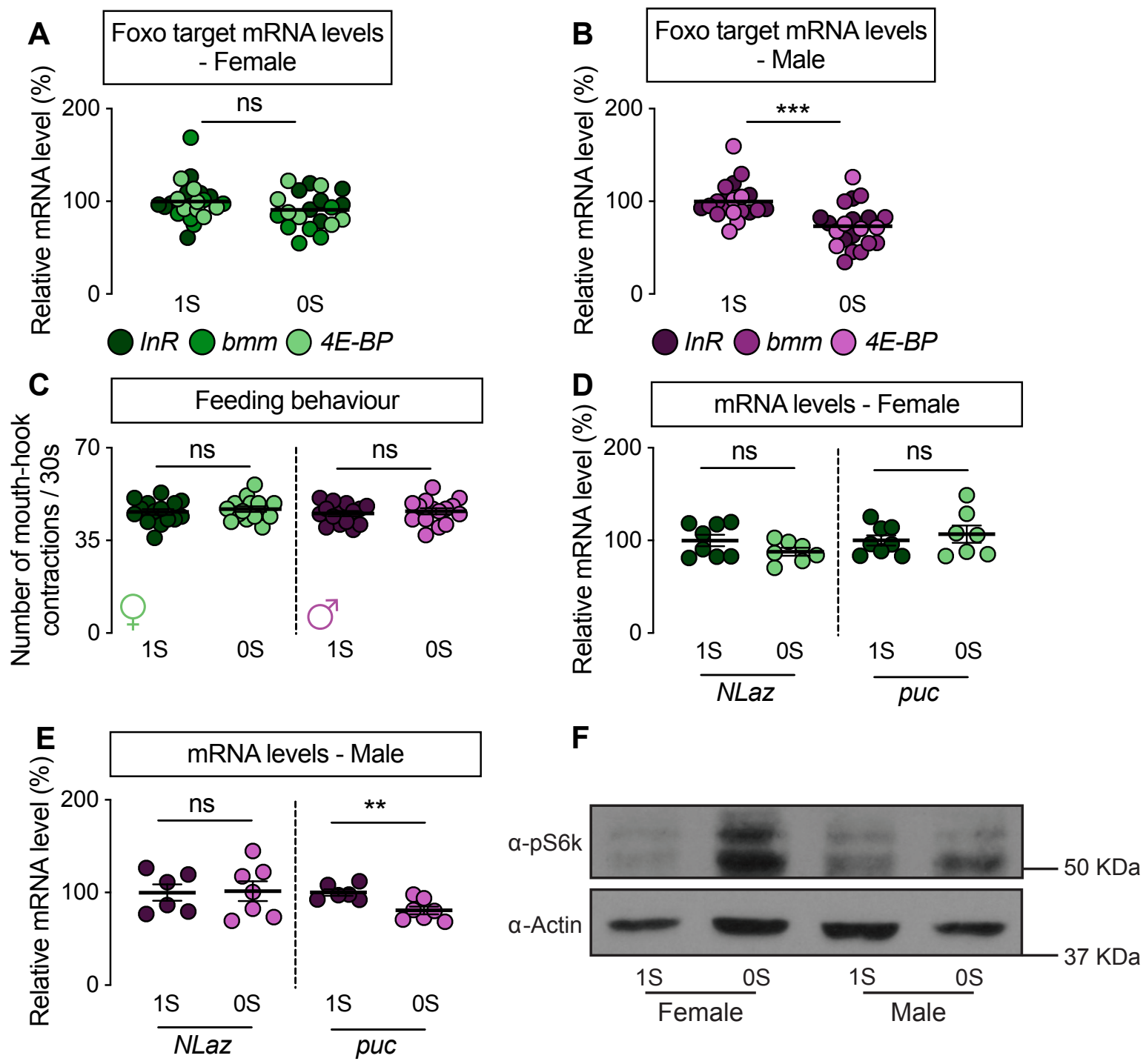

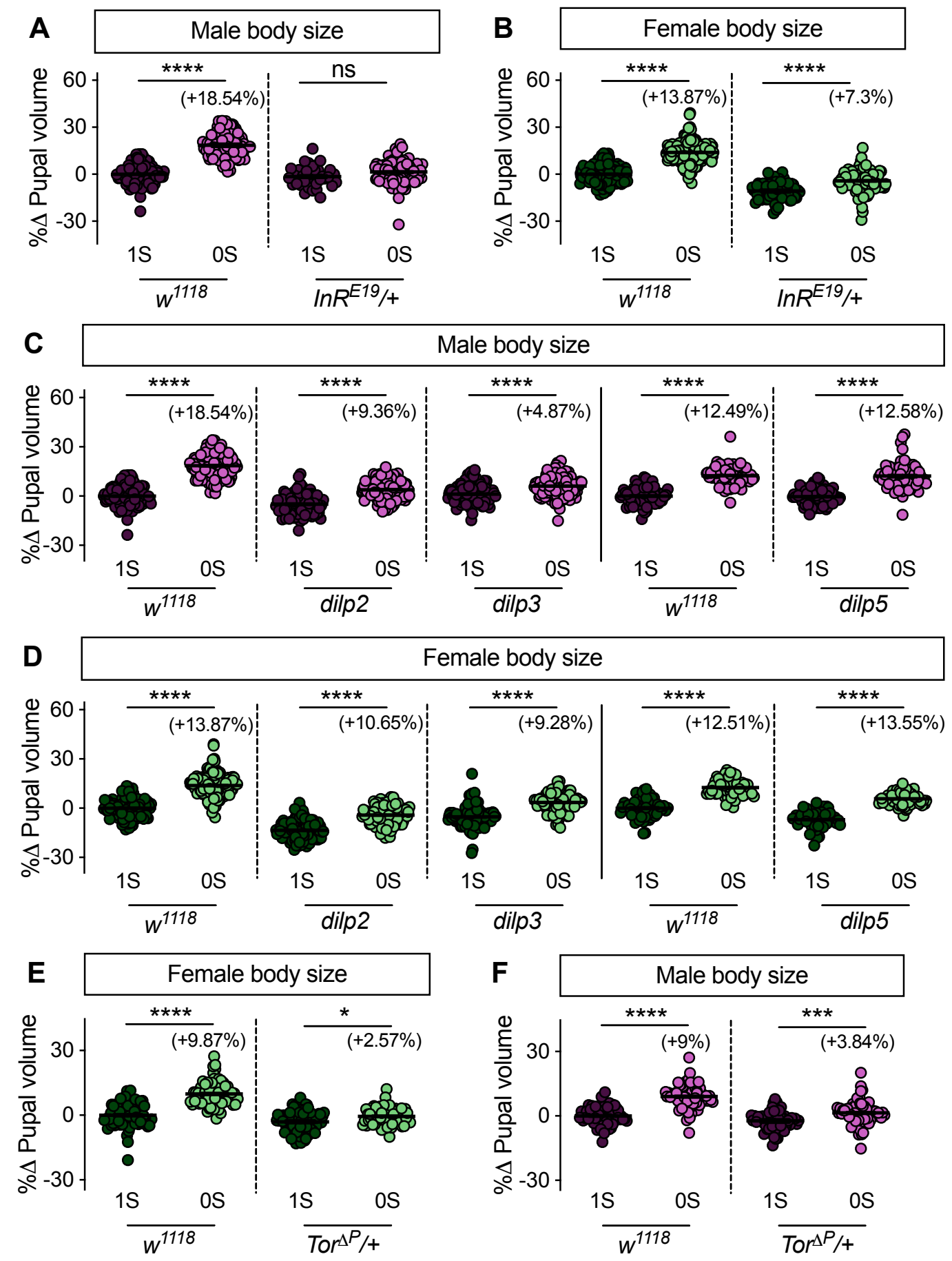
Figure 4.
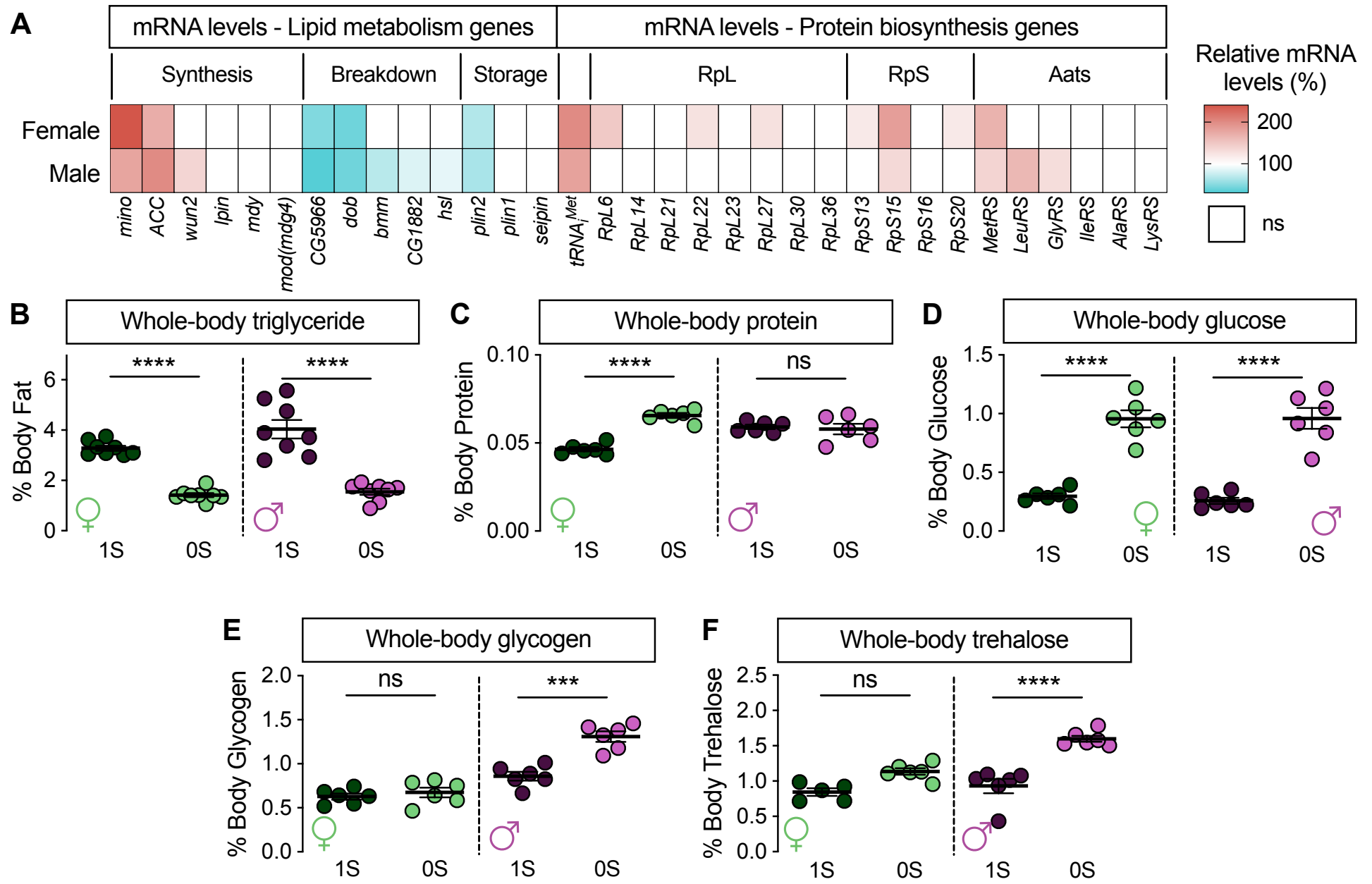
Figure S1.

A

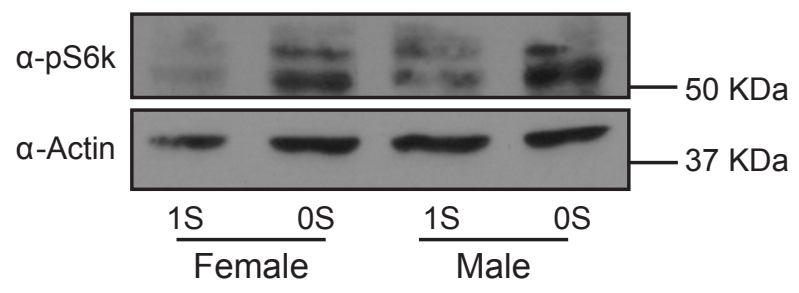

